\begin{tabular}{|c|l|}
\hline Title & In-plane nuclear field formation investigated in single self-assembled quantum dots \\
\hline Author(s) & Y amamoto, S.; Matsusaki, R.; Kaji, R.; A dachi, S. \\
\hline Citation & $\begin{array}{l}\text { Physical Review B, 97(7), 075309 } \\
\text { https://doi.org/10.1103/PhysRevB.97.075309 }\end{array}$ \\
\hline Issue Date & 2018-02-26 \\
\hline Doc URL & http://hdl.handle.net/2115/68854 \\
\hline Rights & @2018A American Physical Society \\
\hline Type & article \\
\hline File Information & PhysRevB.97.075309.pdf \\
\hline
\end{tabular}

Instructions for use 


\title{
In-plane nuclear field formation investigated in single self-assembled quantum dots
}

\author{
S. Yamamoto, ${ }^{*}$ R. Matsusaki, R. Kaji, and S. Adachi ${ }^{\dagger}$ \\ Division of Applied Physics, Hokkaido University, N13 W8, Kitaku, Sapporo 060-8628, Japan
}

(Received 2 October 2017; revised manuscript received 15 February 2018; published 26 February 2018)

\begin{abstract}
We studied the formation mechanism of the in-plane nuclear field in single self-assembled $\mathrm{In}_{0.75} \mathrm{Al}_{0.25} \mathrm{As} / \mathrm{Al}_{0.3} \mathrm{Ga}_{0.7} \mathrm{As}$ quantum dots. The Hanle curves with an anomalously large width and hysteretic behavior at the critical transverse magnetic field were observed in many single quantum dots grown in the same sample. In order to explain the anomalies in the Hanle curve indicating the formation of a large nuclear field perpendicular to the photo-injected electron spin polarization, we propose a new model based on the current phenomenological model for dynamic nuclear spin polarization. The model includes the effects of the nuclear quadrupole interaction and the sign inversion between in-plane and out-of-plane components of nuclear $\mathrm{g}$ factors, and the model calculations reproduce successfully the characteristics of the observed anomalies in the Hanle curves.
\end{abstract}

DOI: 10.1103/PhysRevB.97.075309

\section{INTRODUCTION}

The study of nuclear spin physics in semiconductor quantum dots (QDs) is an active research field currently. This is because the role of hyperfine interaction (HFI), which is the magnetic interaction between a localized electron and the lattice nuclei, is drastically enhanced in QD structures compared with those in bulks and quantum wells due to a strong localization of the electron wave function [1-3]. Since it is possible to transfer the angular momentum from light onto nuclei via electron spin, a macroscopic nuclear spin polarization (NSP) which is orders of magnitude larger than the value in thermal equilibrium can be generated actually at cryogenic temperatures, and in turn, the resultant nuclear field (Overhauser field, $\boldsymbol{B}_{\mathrm{n}}$ ) up to a few Teslas affects the electron spin dynamics significantly [4-10]. Because the lattice nuclei act as a reservoir for an optically or electrically injected electron spin, the engineering of nuclear spins such as the optical manipulation of the NSP not only leads to the potential applications but also opens up a new spin physics.

The dynamics of NSP is determined by the environment to which the nuclei are exposed, such as presences of an external magnetic field and/or an unpaired electron in a QD and dipoledipole interaction among the neighbor nuclei. In particular, nuclear quadrupole interaction (QI), which originates from the coupling of a nuclear spin with $I>1 / 2$ to the electric field gradients (EFG) [11], has received a lot of attention recently. Since the lattice strain is used as a driving force for the spontaneous formation process of self-assembled QDs (SA-QDs), the residual strain and the resultant large EFG arise in these QDs. Therefore, the impact of QI is expected to gain considerably and to play key roles for various novel phenomena observed in SA-QDs [12-15]. The QI yields the nonequivalent energy splitting depending on the value $\left|I_{q}\right|$, where the subscript $q$ is a quantization axis determined by EFG and the $q$ axis is found to be usually close to the sample

\footnotetext{
*yama-st@eis.hokudai.ac.jp

†adachi-s@eng.hokudai.ac.jp
}

growth axis ( $z$ axis) in the SA-QDs [16,17]. Then, the QI can be treated as an effective field affecting the nuclear spins, the quadrupolar field, and it stabilizes the NSP along the $q$ axis as reported in an ensemble of SA-InP/InGaP QDs [18] and single SA-InAs/GaAs QDs [19].

Recently, the formation of in-plane nuclear field was reported in single SA-InAs/GaAs QDs under quasiresonant excitation by Krebs et al. [15] and nonresonant excitation by Nilsson et al. [20], and it seemed to be related also to QI. The in-plane nuclear field was detected by observing the electron spin depolarization curve in Voigt configuration (i.e., Hanle curve). In their pioneering works, the Hanle curve was distorted drastically from a normal Lorentzian shape in the following respects: a $\sim 20$ times larger width than the one expected from the electron spin lifetime, and the abrupt change in the degree of circular polarization (DCP), and thus, the anomalous Hanle curve has a shape like a circus tent. In addition, the fact that such anomalies in the Hanle curve have not been observed in single droplet GaAs QDs [21] which are free from the internal strain suggests that the strain-induced QI contributes significantly to an anomalous Hanle curve observed in SA-QDs. However, the origin of the anomalies in the Hanle curves has not been revealed entirely. The knowledge of the in-plane nuclear field formation may lead directly to an optical control of the nuclear field direction, and therefore, it is very important.

In this paper, we study the formation mechanism of the in-plane nuclear field via the Hanle effect measurements and model calculations. The anomalously distorted Hanle curves similar to Ref. [15] are observed in single InAlAs QDs, and we show that the anomalies of the Hanle curves can be reproduced qualitatively by a proposed model including the QI and the sign inversion of nuclear $\mathrm{g}$ factors.

\section{QD SAMPLE AND ANOMALOUS HANLE CURVES}

SA- $\operatorname{In}_{0.75} \mathrm{Al}_{0.25} \mathrm{As} / \mathrm{Al}_{0.3} \mathrm{Ga}_{0.7} \mathrm{As} \mathrm{QDs}$ grown on a (100)GaAs substrate by molecular beam epitaxy were used in this study. The QDs have a lens-shaped profile, and the typical diameter and height were evaluated to be $\sim 20 \mathrm{~nm}$ and $\sim 4 \mathrm{~nm}$, 
respectively, by the atomic force microscopy measurements of a reference uncapped QD layer and the cross-section transmission electron microscope observation. After the fabrication of small mesa structures, the microphotoluminescence ( $\mu \mathrm{PL})$ measurements under the transverse magnetic fields $\left(B_{x}\right)$ up to $1 \mathrm{~T}$ were carried out at $6 \mathrm{~K}$. The QD sample was excited by a cw Ti:sapphire laser tuned to $\sim 730 \mathrm{~nm}$, which corresponds to the transition energy to the foot of the wetting layer of the QDs.

The polarization of an excitation beam was adjusted carefully to the circular polarization by a combination of a linear polarizer, a half wave plate, and a quarter wave plate (QWP). The circularly polarized $\left(\sigma^{+}, \sigma^{-}\right)$PL components were converted to the linearly polarized ones $\left(\pi^{x}, \pi^{y}\right)$ by another QWP inserted into the detection path, and they were displaced spatially from each other by a beam displacer. Each displaced PL component was dispersed by a spectrometer and was focused on a different area of the Si-CCD detector. Therefore, the energy splitting between the $\sigma^{+}$and $\sigma^{-}$components and the DCP of the PL spectra can be acquired by a single exposure process. The details of the experimental apparatus are seen in Ref. [22]. The energy resolution of our measurement system is $\leqslant 5 \mu \mathrm{eV}$ by spectral fitting.

In this paper, we focus on the PL of a positive trion $\left(X^{+}\right)$, and it appears at $\sim 754 \mathrm{~nm}$ at $0 \mathrm{~T}$ in the typical single QD shown in Fig. 1. The $X^{+}$ground state consists of two holes in a spin-singlet state and one electron, and thus, the DCP of this charge state is determined only by the electron spin right before radiative recombination. Here, the DCP is defined as $\rho_{\mathrm{c}}=\left(I^{-}-I^{+}\right) /\left(I^{-}+I^{+}\right)$, where $I^{+(-)}$represents the integrated PL intensity of the $\sigma^{+(-)}$component. The DCP is related directly to the projection of the averaged electron spin onto the sample growth axis, $\left\langle S_{z}\right\rangle$, and the relation $\left\langle S_{z}\right\rangle=\rho_{\mathrm{c}} / 2$ is held for the $X^{+}$case.

Figure 1(a) is an example of the anomalous Hanle curves observed in the SA-InAlAs QD sample. In the figure, the red (blue) curve represents the observed DCP with increasing (decreasing) $B_{x}$ under $\sigma^{-}$excitation, while the dashed curve is an expected normal Hanle curve, which is free from the effect of $B_{\mathrm{n}}$ and has a Lorentzian shape with the full width $2 B_{1 / 2}(\sim 130 \mathrm{mT})$. The half width $B_{1 / 2}$ is given by $\hbar /\left(\mu_{\mathrm{B}}\left|g_{x}^{\mathrm{e}}\right| T_{\mathrm{S}}\right)$, where $\mu_{\mathrm{B}}$ is the Bohr magneton, $\left|g_{x}^{\mathrm{e}}\right|$ is the in-plane electron $\mathrm{g}$ factor, and $T_{\mathrm{s}}$ is the electron spin lifetime. The $B_{1 / 2}$ is evaluated as a typical value for the studied QD sample by using the measured $\left|g_{x}^{\mathrm{e}}\right|=0.35$ (see Appendix A) and $T_{\mathrm{s}}=1 /\left(1 / \tau_{\mathrm{r}}+1 / \tau_{\mathrm{s}}\right)=0.5 \mathrm{~ns}$. It is known that InAlAs/Al(Ga)As QDs have the complex band structure depending on the $\mathrm{Al}$ concentration and the QD size [23]. In the studied $\operatorname{In}_{0.75} \mathrm{Al}_{0.25} \mathrm{As} / \mathrm{Al}_{0.3} \mathrm{Ga}_{0.7} \mathrm{As}$ QDs, the lowest electron level appears always in the $\Gamma$ valley, and the resultant direct QD exciton has a short recombination time $\tau_{\mathrm{r}} \sim 1.0 \mathrm{~ns}$ as evaluated from the time-resolved PL measurements [24]. Also, the electron spin relaxation time $\tau_{\mathrm{s}}$ is found to be $\sim \tau_{\mathrm{r}}$ by nuclear field fluctuation measurements $[22,25]$.

As clearly shown, the observed curves have quite larger widths than the expected one. Further, the DCP changes suddenly at the critical field $\left|B_{x}^{\mathrm{c}}\right| \sim 0.8 \mathrm{~T}$, and $\left|B_{x}^{\mathrm{c}}\right|$ is different depending on the sweep direction of $B_{x}$; for the red (blue) line, the outer $B_{x}^{\mathrm{c}}=+0.840 \mathrm{~T}(-0.790 \mathrm{~T})$ and the inner $B_{x}^{\mathrm{c}}=-0.720 \mathrm{~T}(+0.773 \mathrm{~T})$ in Fig. 1(a). Such anomalous characters were observed in not only a specific QD but also

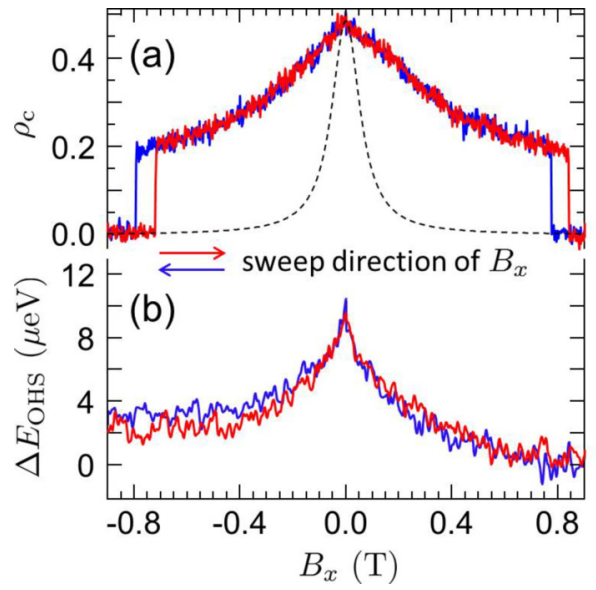

FIG. 1. (a) The anomalous Hanle curves observed in the SAInAlAs QD. The red (blue) curve represents the DCP of $X^{+}$PL with increasing (decreasing) $B_{x}$. The dotted curve is the normal Hanle curve expected with typical values of spin lifetime and $\mathrm{g}$ factor of electron. (b) The observed Overhauser shift as a function of $B_{x}$, which is used as a measure of $\left|B_{\mathrm{n}, z}\right|$.

all the QDs we measured in the same sample (not shown here). In the work by Krebs et al. [15], the Hanle curves with the similar anomalies were observed in single SA-InAs/GaAs QDs, where the bistable behavior of a nuclear field in the $x$ direction $B_{\mathrm{n}, x}$ and a compensation of $B_{x}$ by $B_{\mathrm{n}, x}$ were confirmed unambiguously.

The observed energy splittings between the $\sigma^{+}$and $\sigma^{-}$ PL components are plotted in Fig. 1(b). The $z$ component of the nuclear field, $B_{\mathrm{n}, z}$, can be estimated by the energy splitting detected in the $\left(\sigma^{+}, \sigma^{-}\right)$basis, which is termed the Overhauser shift, $\Delta E_{\mathrm{OHS}}$. As seen in the figure, the maximal value of $\Delta E_{\mathrm{OHS}}$ of $\sim 10 \mu \mathrm{eV}$ appears at $B_{x}=0 \mathrm{~T}$, and it corresponds to the $B_{\mathrm{n}, z}$ of $-0.5 \mathrm{~T}$ by considering the relation $B_{\mathrm{n}, z}=\Delta E_{\mathrm{OHS}} /\left(g_{z}^{\mathrm{e}} \mu_{\mathrm{B}}\right)$, where $g_{z}^{\mathrm{e}}$ is the electron $\mathrm{g}$ factor in the $z$ direction. $\Delta E_{\mathrm{OHS}}$ reduces gradually with increasing $\left|B_{x}\right|$ and approaches almost zero around $\left|B_{x}^{\mathrm{c}}\right|$. Further, the observed $\Delta E_{\mathrm{OHS}}$ is slightly asymmetric with respect to the sign in $B_{x}$. The asymmetry of $\Delta E_{\mathrm{OHS}}$ does not depend on the sweep direction of $B_{x}$, and the pattern of $\left|\Delta E_{\mathrm{OHS}}\right|$ reverses with respect to $B_{x}$ under the opposite helicity excitation ( $\sigma^{+}$pump). The similar asymmetry in $\Delta E_{\mathrm{OHS}}$ is observed in several single QDs grown in the same sample, but a few QDs show the symmetrical $\Delta E_{\mathrm{OHS}}$. Therefore, we consider that the effect of unintentional tilted sample mounting is not responsible for the asymmetric $\Delta E_{\mathrm{OHS}}$. The physical origin of the asymmetry has not been figured out at present, and further experimental investigations are necessary. Finally, as discussed later in Sec. IV, the Zeeman splitting by the total magnetic field in the outside of $\left|B_{x}^{\mathrm{c}}\right|>\sim 0.8 \mathrm{~T}$ should be determined dominantly by the externally applied field $B_{x}$, and $\Delta E_{\mathrm{OHS}}$ and $B_{\mathrm{n}, x}$ should be nearly zero. However, as shown in Fig. 1(b), $\Delta E_{\mathrm{OHS}}$ has nonzero values in $B_{x}<B_{x}^{\mathrm{c}} \sim-0.8 \mathrm{~T}$. This disagreement is considered to arise from the shortage of our energy resolution.

The set of the electron $\mathrm{g}$ factor of this single QD $\left(g_{x}^{\mathrm{e}}, g_{y}^{\mathrm{e}}\right.$, $g_{z}^{\mathrm{e}}$ ) is described in Appendix A, and it is required for the evaluation of the nuclear field and the model calculations in the next section. 


\section{MODEL CALCULATIONS}

In this section, we propose a dynamics model of a coupled electron-nuclear spin system in order to reproduce the observed anomalous Hanle curves shown in the previous section.

The evolution of an electron spin polarization $\langle\boldsymbol{S}\rangle$ can be described by the Bloch equation:

$$
\frac{d\langle\boldsymbol{S}\rangle}{d t}=\frac{\bar{g}_{\mathrm{e}} \mu_{\mathrm{B}}}{\hbar} \boldsymbol{B}_{\mathrm{T}}^{(\mathrm{e})} \times\langle\boldsymbol{S}\rangle-\frac{\langle\boldsymbol{S}\rangle-\boldsymbol{S}_{0}}{T_{\mathrm{S}}},
$$

where $\bar{g}_{\mathrm{e}}$ is the electron $\mathrm{g}$ tensor, $\boldsymbol{B}_{\mathrm{T}}^{(\mathrm{e})}$ is an effective magnetic field seen by a QD electron, and $\boldsymbol{S}_{0}=\left(0,0, S_{0}\right)$ is the average electron spin polarization in the absence of $\boldsymbol{B}_{\mathrm{T}}^{(\mathrm{e})}$. The first and the second terms in the right-hand side represent the Larmor precession and the electron spin decay with a characteristic time $T_{\mathrm{s}}$, respectively. The steady state solution of Eq. (1) gives a Hanle curve according to the relation $\rho_{\mathrm{c}}=2\left\langle S_{z}\right\rangle$ (see Appendix B). Since the nuclear field $\boldsymbol{B}_{\mathrm{n}}(\propto\langle\boldsymbol{I}\rangle$ : NSP $)$ as well as the externally applied field $\boldsymbol{B}_{\text {ext }}$ contributes to $\boldsymbol{B}_{\mathrm{T}}^{(\mathrm{e})}$ $\left(\equiv \boldsymbol{B}_{\text {ext }}+\boldsymbol{B}_{\mathrm{n}}\right)$, it is essential to consider the spin dynamics of nuclei. In this work, the external field is applied in the sample growth plane, and thus, it can be written as $\boldsymbol{B}_{\text {ext }}=\left(B_{x}, 0,0\right)$.

As widely accepted, the contact-type HFI includes the flip-flop term between an electron and nuclear spins, i.e., $\propto\left(\hat{I}_{+} \hat{S}_{-}+\hat{I}_{-} \hat{S}_{+}\right)$, and it allows the spin transfer from the optically-injected electron to the lattice nuclei system [1]. The dynamics of the NSP component $\left\langle I_{k}\right\rangle(k=x, y, z)$ follows the phenomenological equation $[2,3]$ :

$$
\frac{d\left\langle I_{k}\right\rangle}{d t}=\frac{1}{T_{\mathrm{NF}, k}}\left[Q\left(\left\langle S_{k}\right\rangle-\left\langle S_{k}^{\mathrm{eq}}\right\rangle\right)-\left\langle I_{k}\right\rangle\right]-\frac{1}{T_{\mathrm{ND}, k}}\left\langle I_{k}\right\rangle,
$$

where $Q=\tilde{I}(\tilde{I}+1) /[S(S+1)]$ is a numerical constant of the momentum conversion, $\left\langle S_{k}^{\mathrm{eq}}\right\rangle$ is the electron spin polarization at thermal equilibrium, and $1 / T_{\mathrm{ND}, k}$ is the relaxation rate of $\left\langle I_{k}\right\rangle$. The NSP formation rate $1 / T_{\mathrm{NF}, k}$ in the $k$ direction depends on $\left\langle I_{k}\right\rangle$ itself, and it is given by

$$
\frac{1}{T_{\mathrm{NF}, k}}=2 f_{\mathrm{e}} \tau_{\mathrm{c}}\left(\frac{\tilde{A}_{k}}{N \hbar}\right)^{2} /\left\{1+\left[g_{k}^{\mathrm{e}} \mu_{\mathrm{B}}\left(B_{\mathrm{ext}, k}+B_{\mathrm{n}, k}\right) \tau_{\mathrm{c}} / \hbar\right]^{2}\right\},
$$

where $f_{\mathrm{e}}\left(0 \leqslant f_{\mathrm{e}} \leqslant 1\right)$ is a filling factor representing the occupation of a QD by an unpaired electron spin, $\tau_{\mathrm{c}}$ is a correlation time of HFI, $N$ is the number of nuclei related to the interaction, $\tilde{A}_{k}$ is the averaged coupling constant of HFI, and $B_{\mathrm{n}, k}=\tilde{A}_{k} I_{k} /\left(g_{k}^{\mathrm{e}} \mu_{\mathrm{B}}\right)$ is the $k$ component of the nuclear field. This spin dynamics model has explained successfully the experimental observations about $\left\langle I_{z}\right\rangle$ and thus the behavior of $B_{\mathrm{n}, z}$ in the previous studies [6-10].

Moreover, it is necessary to consider the effective magnetic field experienced by nuclear spins, $\boldsymbol{B}_{\mathrm{T}}^{(\mathrm{n})}$ explicitly. As is the case with $\boldsymbol{B}_{\mathrm{T}}^{(\mathrm{e})}, \boldsymbol{B}_{\mathrm{T}}^{(\mathrm{n})}$ is determined by the sum of the external field and the Knight field $\boldsymbol{B}_{\mathrm{e}}$, i.e., $\boldsymbol{B}_{\mathrm{ext}}+\boldsymbol{B}_{\mathrm{e}}$. Here, $\boldsymbol{B}_{\mathrm{e}}$ refers to the effective field caused by the electron spin polarization and is given by $\boldsymbol{B}_{\mathrm{e}}=-f_{\mathrm{e}} \tilde{A}\langle\boldsymbol{S}\rangle /\left(\bar{g}_{\mathrm{n}} \mu_{\mathrm{N}} N\right)$, where $\bar{g}_{\mathrm{n}}$ is the nuclear $\mathrm{g}$ tensor and $\mu_{\mathrm{N}}$ is the nuclear magneton. The introduction of $\boldsymbol{B}_{\mathrm{e}}$ explains the modifications of Hanle curves [1] which appear in the very low magnetic field region $\left(B_{\mathrm{ext}} \lesssim 0.1 \mathrm{~T}\right)$ as shown later [Figs. 3(c) and 3(d)]. However, it is not possible to describe the observed anomalous Hanle curve with a quite large width (e.g.,
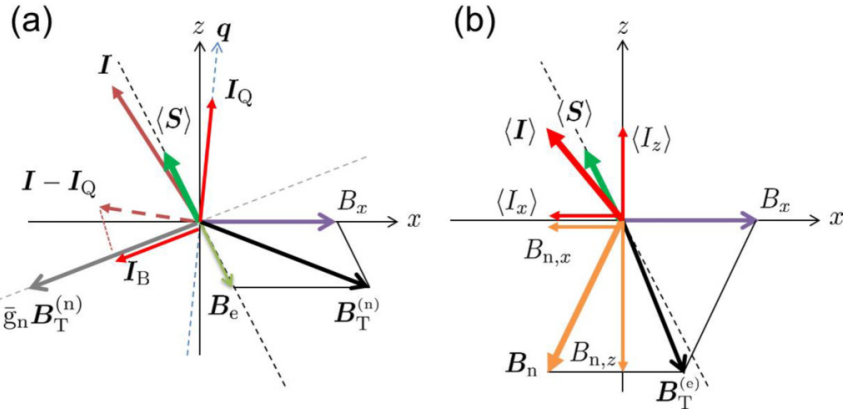

FIG. 2. Schematics of the spin polarizations and the resultant effective fields. Here, the isotropic electron $g$ factor $\left(g_{k}^{\mathrm{e}}<0\right)$ is assumed. For generality, the unit vector $\boldsymbol{q}$ of the QI axis is tilted slightly from the $z$ axis. (a) A portion of the HFI-induced $\boldsymbol{I}$ is protected by QI and the other one is preserved by the actual effective field $\bar{g}_{\mathrm{n}} \boldsymbol{B}_{\mathrm{T}}^{(\mathrm{n})}: \boldsymbol{I}_{\mathrm{Q}}$ and $\boldsymbol{I}_{\mathrm{B}}$. Since $g_{x}^{\mathrm{n}}<0$ and $g_{z}^{\mathrm{n}}>0$ are assumed here, the $\bar{g}_{\mathrm{n}} \boldsymbol{B}_{\mathrm{T}}^{(\mathrm{n})}$ is depicted to be opposite in the $x$ direction to $\boldsymbol{B}_{\mathrm{T}}^{(\mathrm{n})}$ while the $z$ component is unchanged. (b) The protected NSP $\langle\boldsymbol{I}\rangle\left(=\boldsymbol{I}_{\mathrm{Q}}+\boldsymbol{I}_{\mathrm{B}}\right)$ induces $\boldsymbol{B}_{\mathrm{n}}$ eventually. Because of $g_{x}^{\mathrm{n}}<0, g_{z}^{\mathrm{n}}>0$, and $g_{k}^{\mathrm{e}}<0, B_{\mathrm{n}, x}$ is directed in parallel to $\left\langle I_{x}\right\rangle$ while $B_{\mathrm{n}, z}$ is antiparallel to $\left\langle I_{z}\right\rangle$.

$\left|B_{x}^{\mathrm{c}}\right| \sim 0.8 \mathrm{~T}$ in Fig. 1(a) and the results in Ref. [15]) in this framework. Therefore, we improve the conventional model by introducing the effects of QI.

One of the significant improvements is the NSP stabilization due to QI. It should be noted that the HFI-induced NSP $I$ is not collinear with the electron spin polarization $\langle\boldsymbol{S}\rangle$ as depicted in Fig. 2(a) because of $1 / T_{\mathrm{NF}, x} \neq 1 / T_{\mathrm{NF}, z}$, while it becomes collinear with $\langle\boldsymbol{S}\rangle$ according to Eq. (2) if $1 / T_{\mathrm{NF}, k}$ is isotropic. Here, we assume that $\boldsymbol{I}$ along the principal axis of QI is preserved partially with a ratio $r_{\mathrm{Q}}\left(0<r_{\mathrm{Q}} \leqslant 1\right)$. Hence the QI-preserving component $\boldsymbol{I}_{\mathrm{Q}}$ is given as

$$
\boldsymbol{I}_{\mathrm{Q}}=r_{\mathrm{Q}}(\boldsymbol{I} \cdot \boldsymbol{q}) \boldsymbol{q},
$$

where $\boldsymbol{q}$ is a unit vector along with the principal axis of QI.

The other component (i.e., $\boldsymbol{I}-\boldsymbol{I}_{\mathrm{Q}}$ ) is affected by the effective magnetic field, and its projection onto $\bar{g}_{\mathrm{n}} \boldsymbol{B}_{\mathrm{T}}^{(\mathrm{n})}$ termed $\boldsymbol{I}_{\mathrm{B}}$ is also preserved as follows:

$$
\boldsymbol{I}_{\mathrm{B}}=\frac{\left(\boldsymbol{I}-\boldsymbol{I}_{\mathrm{Q}}\right) \cdot \bar{g}_{\mathrm{n}} \boldsymbol{B}_{\mathrm{T}}^{(\mathrm{n})}}{\left|\bar{g}_{\mathrm{n}} \boldsymbol{B}_{\mathrm{T}}^{(\mathrm{n})}\right|} \frac{\bar{g}_{\mathrm{n}} \boldsymbol{B}_{\mathrm{T}}^{(\mathrm{n})}}{\left|\bar{g}_{\mathrm{n}} \boldsymbol{B}_{\mathrm{T}}^{(\mathrm{n})}\right|} .
$$

The term $\bar{g}_{\mathrm{n}} \boldsymbol{B}_{\mathrm{T}}^{(\mathrm{n})}$ is the effective magnetic field experienced actually by the nuclei and is convenient for the case with anisotropic $\bar{g}_{\mathrm{n}}$. Eventually as shown in Fig. 2(b), only the sum of $\boldsymbol{I}_{\mathrm{Q}}$ and $\boldsymbol{I}_{\mathrm{B}},\langle\boldsymbol{I}\rangle$ survives and acts as a nuclear field according to the following relation:

$$
\boldsymbol{B}_{\mathrm{n}}=\frac{\tilde{A}}{\bar{g}_{\mathrm{e}} \mu_{\mathrm{B}}}\left(\boldsymbol{I}_{\mathrm{Q}}+\boldsymbol{I}_{\mathrm{B}}\right) .
$$

In the following model calculations, we assume that the ratio $r_{\mathrm{Q}}$ in Eq. (4) depends on the applied transverse field as follows:

$$
r_{\mathrm{Q}}\left(B_{x}\right)=\frac{r_{0}}{1+\left(B_{x} / B_{\mathrm{Q}}\right)^{2}},
$$

where $B_{\mathrm{Q}}$ is the quadrupolar field. Note that the $B_{\mathrm{Q}}$ cannot induce the spin precession. The parameter $r_{0}$ is an amplitude of the Lorentzian-type function, and the condition $r_{0}=1$ is 
used in the calculations for simplicity. The efficiency of the NSP stabilization is supposed to decrease with increasing $\left|B_{x}\right|$ if the $\boldsymbol{q}$ axis is almost perpendicular to $B_{x}$. This is because the relative strength of QI to $B_{x}$ reduces with increasing $\left|B_{x}\right|$ and the NSP component perpendicular to $B_{x}$ is easy to relax. In this single InAlAs QD, the magnitude of $B_{\mathrm{Q}}$ is estimated to be $280 \pm 40 \mathrm{mT}$ from the independent experiments [22].

Further, we assume an anisotropic nature in nuclear $\mathrm{g}$ tensor, that is, the sign of $g_{x(y)}^{\mathrm{n}}$ is opposite to that of $g_{z}^{\mathrm{n}}$. Thus, the effective field $\bar{g}_{\mathrm{n}} \boldsymbol{B}_{\mathrm{T}}^{(\mathrm{n})}$ is opposite to $\boldsymbol{B}_{\mathrm{T}}^{(\mathrm{n})}$ in the $x$ direction [Fig. 2(a)]. Also, because $B_{\mathrm{n}, k}$ in Eq. (6) includes the HFI coupling constant $\tilde{A}_{k}\left(\propto g_{k}^{\mathrm{n}}\right)$ and $g_{k}^{\mathrm{e}}$, the direction of the resultant $B_{\mathrm{n}, k}$ is determined by the signs of $g_{k}^{\mathrm{n}}$ and $g_{k}^{\mathrm{e}}$. Therefore, $B_{\mathrm{n}, x}$ is depicted to be parallel to $\left\langle I_{x}\right\rangle$ while $B_{\mathrm{n}, z}$ is antiparallel to $\left\langle I_{z}\right\rangle$ [Fig. 2(b)]. Such a sign inversion of the nuclear g factors is required to achieve the compensation of the external field $B_{x}$ by $B_{\mathrm{n}, x}$ that increases by a flip-flop process of HFI.

Figure 3 highlights the impacts of the NSP stabilization by $\mathrm{QI}$ and the sign inversions of $\mathrm{g}$ factors. The former represents the presence or absence of $\boldsymbol{I}_{\mathrm{Q}}$, i.e., $r_{\mathrm{Q}} \neq 0$ or $r_{\mathrm{Q}}=0$. The latter includes the two types of sign inversions: the sign inversion between the in-plane and the out-of-plane components of $g^{\text {n }}$ [that is, the anisotropy in the g tensor, shown in Figs. 3(a) and3(b)] and the one between the isotropic $g^{\mathrm{e}}$ and $g^{\mathrm{n}}$ [as shown in Figs. 3(c) and3(d)]. In the figure, the relaxation times of the NSP $T_{\mathrm{ND}, k}(k=x, y, z)$ are assumed to be equivalent, and all the other parameters are identical in (a)-(d) [26].

Replacing $g_{x(y)}^{\mathrm{n}} \cdot g_{z}^{\mathrm{n}}$ with $g_{x(y)}^{\mathrm{e}} \cdot g_{z}^{\mathrm{e}}$ gives the similar calculated results in Figs. 3(a) and 3(b). However, as seen in Appendix A, the $\mathrm{g}$ factor of the conduction electron is isotropic in magnitude, and $g_{x(y)}^{\mathrm{e}}$ and $g_{z}^{\mathrm{e}}$ should have the same sign. In contrast, the nuclear $\mathrm{g}$ factor is considered to have a large anisotropy because of QI as discussed later. Therefore, it is likely that the requirement of the sign inversion in the in-plane and out-of-plane $\mathrm{g}$ factors of this study could be attributed to the nuclear $\mathrm{g}$ factors. Also, it should be noted that the possibility of observing the anomalous Hanle curve is independent of the sign of the electron $\mathrm{g}$ factor.

In Figs. 3(a) and 3(b), we set $g_{z}^{\mathrm{n}}>0$ and the isotropic electron $\mathrm{g}$ factors with a negative sign. In the case of (a) $\left(r_{\mathrm{Q}} \neq 0\right.$ and $g_{x}^{\mathrm{n}} \cdot g_{z}^{\mathrm{n}}<0$ ), the Hanle curve shows a much larger full width than $2 B_{1 / 2}$ indicated by the dotted line. The extension of the width is induced by the compensation of $B_{x}$ by $B_{\mathrm{n}, x}$ that follows the change in $B_{x}$ depicted in Fig. 2(b), and therefore, the bistable (hysteretic) behavior appears. On the other hand, the effect of nonzero $r_{\mathrm{Q}}$ is strongly reduced in Fig. 3(b) since $B_{\mathrm{n}, x}$ enhances the total effective field on the electron spin, and the growth of $B_{\mathrm{n}, x}$ is terminated by high energy cost in the flip-flop process. Therefore, only a slight broadening of the curve occurs.

In the case of $r_{\mathrm{Q}}=0$ [(c) and (d)], the Hanle curves are classified by the sign of $g_{k}^{\mathrm{e}} \cdot g_{k}^{\mathrm{n}}$ since $g_{k}^{\mathrm{n}}$ should be isotropic in the QI-free system. As clearly shown, the effect of tilting $\boldsymbol{B}_{\mathrm{n}}$ by the applied field $B_{x}$ is reproduced as discussed in Ref. [1], where the sign of the amplification factor $\kappa\left(\propto g_{k}^{\mathrm{e}} \cdot g_{k}^{\mathrm{n}}\right)$ determines whether the compensation or the enhancement of $B_{x}$ by $B_{\mathrm{n}, x}$ occurs, as depicted in (c) and (d), respectively. The W-shaped dip (i.e., recovery of $\left\langle S_{z}\right\rangle$ ) in (c) may correspond to the actual observations in GaAlAs bulk [1] and single droplet GaAs/AlGaAs QDs [21]. Since an emerged $B_{\mathrm{n}, x}$ is small and

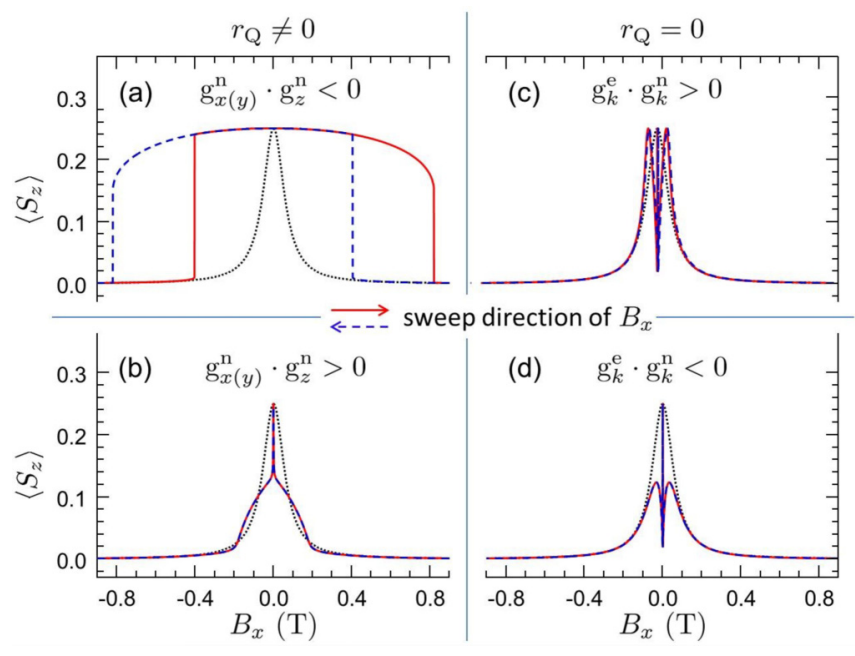

FIG. 3. The typical calculated $\left\langle S_{z}\right\rangle$ depending on QI and the sign of $g_{x}^{\mathrm{n}}$. Here, the isotropic $g_{k}^{\mathrm{e}}$ is assumed, and $g_{z}^{\mathrm{n}}$ is set to be positive $\left(g_{z}^{\mathrm{n}}>0\right)$. (a) $r_{\mathrm{Q}} \neq 0, g_{x}^{\mathrm{n}}<0, g_{k}^{\mathrm{e}}<0,(\mathrm{~b}) r_{\mathrm{Q}} \neq 0, g_{x}^{\mathrm{n}}>0, g_{k}^{\mathrm{e}}<0$. The principal axis of QI is set along the $z$ axis. In the case of $r_{\mathrm{Q}}=0$ [(c) and (d)], $g_{k}^{\mathrm{n}}$ should be isotropic, and the Hanle curves are classified by the sign of $g_{k}^{\mathrm{e}} \cdot g_{k}^{\mathrm{n}}:$ (c) $g_{k}^{\mathrm{e}} \cdot g_{k}^{\mathrm{n}}>0$, (d) $g_{k}^{\mathrm{e}} \cdot g_{k}^{\mathrm{n}}<0$. Other parameters are the same in all cases [26]. The dotted line represents a Lorentzian Hanle curve with the typical InAlAs QD parameters.

nearly constant, the modification of Hanle curve occurs within a low $B_{x}$ region $(\leqslant 0.1 \mathrm{~T})$, and therefore, the depolarization is converging quickly to the tails of a normal Lorentzian curve regardless of the sweep direction of $B_{x}$.

\section{DISCUSSION}

First, we discuss the effect of QI and anisotropic nature of nuclear $\mathrm{g}$ factor. Figure 4 shows the energy splitting of the states for a single As nucleus $(I=3 / 2)$, for example, under the (a) longitudinal and (b) transverse magnetic fields. The vertical and horizontal axes are normalized by $\hbar \omega_{\mathrm{Q}}$ and $B_{\mathrm{Q}}$, respectively, and the $q$ axis lies along the $z$ axis for simplicity. Here, $\hbar \omega_{\mathrm{Q}}$ is a quadrupolar splitting and appears as an energy separation between the doublets labeled $| \pm 1 / 2\rangle_{q}$ and $| \pm 3 / 2\rangle_{q}$ at $0 \mathrm{~T}$. The QI and Zeeman Hamiltonians are described in Appendix C.

The Zeeman-split states in the absence of QI depicted by the dashed lines evolve linearly on the magnetic field, and the splitting is always equivalent and is independent of the applied direction of the field, i.e., $g^{\mathrm{n}}$ of each state is isotropic. In contrast, in the presence of QI, the orthogonal setting of the $q$ axis and $B_{x}$ in Voigt geometry causes some states to be mixed as the system evolves while a simple energy shift by the quadrupolar splitting occurs in Faraday geometry [27]. The effect of the quadrupolar splitting is reducing with increasing $B_{x}$ and each state labeled $|n\rangle(n=1-4)$ is converging to the Zeeman-split pure states $|m\rangle_{x}(m= \pm 1 / 2, \pm 3 / 2)$ in the $x$ basis. However, due to the quadrupolar effect, the states $|4\rangle$ and $|3\rangle$ are insensitive to $B_{x}$ until a large $B_{x}$. Similar insensitivity to $B_{x}$ is seen in the states with $\left|m_{q}\right| \geqslant 3 / 2$ of $\operatorname{In}(I=9 / 2)$ and $\mathrm{Al}(I=5 / 2)$ nuclei, and the state with a large $\left|m_{q}\right|$ becomes more insensitive to $B_{x}$. 

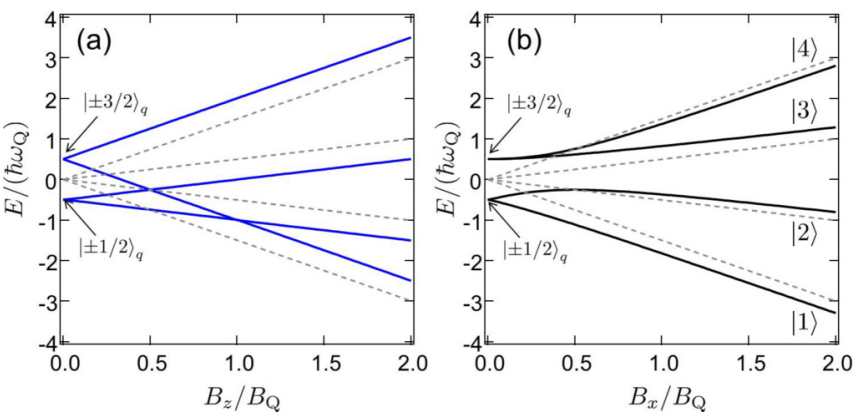

FIG. 4. The eigenstates of a single As nucleus $(I=3 / 2)$ under QI and Zeeman interaction in (a) Faraday and (b) Voigt geometries. The principal axis $\boldsymbol{q}$ is assumed to be along the growth axis. The dashed lines indicate the Zeeman-split states under no QI.

This effect by the quadrupolar splitting induces an anisotropic property to the nuclear $\mathrm{g}$ factor. For the states with $\left|m_{q}\right|=3 / 2$, the nuclear $\mathrm{g}$ factor perpendicular to the $q$ axis $\left(g_{\perp}^{\mathrm{n}}\right)$ is reduced to be nearly zero in a low $B_{x}$ region while the parallel component $\left(g_{\|}^{\mathrm{n}}\right)$ has a finite value although the nuclear $\mathrm{g}$ factor of the states with $\left|m_{q}\right|=1 / 2$ is isotropic. Such a nuclear $\mathrm{g}$ factor anisotropy induced by QI is discussed in $\mathrm{Ga}_{0.76} \mathrm{Al}_{0.24} \mathrm{As}$ bulk [1]. In GaAlAs bulk, the EFG, hence the QI arises from the random alloying (the partial replacement of $\mathrm{Ga}$ atoms in the $\mathrm{GaAs}$ lattice by $\mathrm{Al}$ ) and affects the shape of the Hanle curve. Since the principal axes of QI lie along the Al-As bonds (the third-order axes $\langle 111\rangle$ ), the shape of the Hanle curve depends strongly on the azimuth angle of $B_{x}$ from the crystallographic [110] axis. In contrast, a drastic deformation of Hanle curve in a SA-InAlAs QD as seen in Fig. 1 is insensitive to the azimuth angle of $B_{x}$ (not shown here). This suggests that the residual strain in SA-InAlAs QDs has a larger contribution to QI than the random alloying, and the resultant principal axis of QI is nearly along the $z$ axis.

One of smart modeling taking into account a anisotropic nature of nuclear $\mathrm{g}$ factors is a divided treatment of the nuclear field originated from the states with $\pm 1 / 2$ (dipolar component) and $\left|m_{q}\right| \geqslant 3 / 2$ (quadrupolar component). Kuznetsova et al. used the method in the analysis of the deformation of W-shaped Hanle curves in InGaAs QD ensemble and revealed that the dipolar component contributed to form the $\mathrm{W}$-shaped structure at the center of the Hanle curve and the quadrupolar component made the wing shapes of the curves [28].

In order to maintain the high DCP under large $\left|B_{x}\right|$ (< $\left|B_{x}^{\mathrm{c}}\right| \sim 0.8 \mathrm{~T}$ ), the in-plane nuclear field has to grow up with compensating $B_{x}$. Under this condition, many nuclear spin states with $\left|m_{q}\right| \geqslant 3 / 2$ of the quadrupolar nuclei contribute to achieve the high NSP corresponding to the large $B_{\mathrm{n}, x}$. As seen in Fig. 4, it should be pointed out that each splitting has a different field dependence, and thus, each state is considered to have a different effective $\mathrm{g}$ factor. Therefore, the divided treatment of the contributions from many nuclear spin states with different $m_{q}$ is difficult to be taken into our present model. Although the rigorous modeling requires us to consider the distribution of effective nuclear $\mathrm{g}$ factors, the incorporation to our phenomenological model is beyond our scope at current stage. As mentioned above, the QI with a (nearly) longitudinal axis brings the anisotropic $g^{\mathrm{n}}$ and the insensitivity to the transverse field. Since the component $\left\langle I_{z}\right\rangle$ relaxes quickly (a)

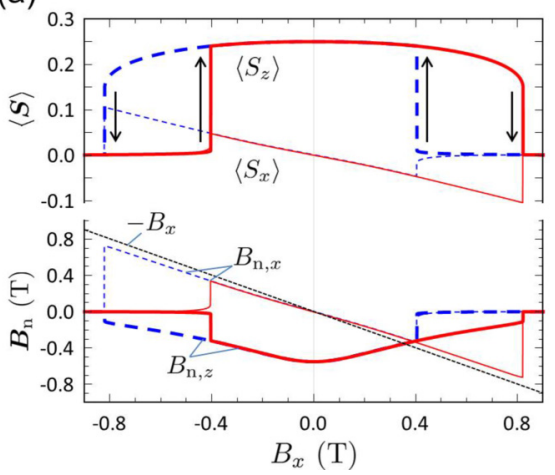

(b)

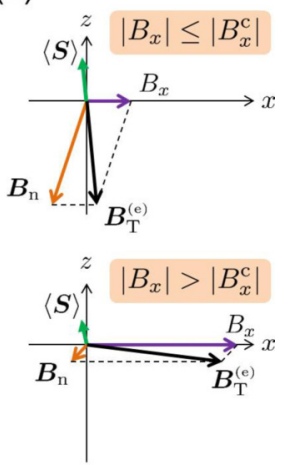

FIG. 5. (a) The calculated $\left\langle S_{z}\right\rangle$ (thick lines) and $\left\langle S_{x}\right\rangle$ (thin lines) in the upper panel, and $B_{\mathrm{n}, z}$ (thick lines) and $B_{\mathrm{n}, x}$ (thin lines) in the lower panel under the conditions $r_{\mathrm{Q}} \neq 0$ and $g_{x}^{\mathrm{n}} \cdot g_{z}^{\mathrm{n}}<0$. The solid (dashed) lines represent the results with increasing (decreasing) $B_{x} .\left\langle S_{y}\right\rangle$ and $B_{\mathrm{n}, y}$ are not shown here for an easy view [26]. (b) Schematics of $\langle\boldsymbol{S}\rangle$ and $\boldsymbol{B}_{\mathrm{n}}$ in the $x-z$ plane corresponding to the case $\left|B_{x}\right| \leqslant\left|B_{x}^{\mathrm{c}}\right|$ (upper panel) and $\left|B_{x}\right|>\left|B_{x}^{\mathrm{c}}\right|$ (lower panel). The complete cancellation relation $B_{\mathrm{n}, x}=-B_{x}$ is indicated by a dotted line.

without the insensitivity to $B_{x}$, this effect can be treated simply by $r_{\mathrm{Q}}$ [Eqs. (4) and (7)] at present avoiding the complexity of the effective nuclear $\mathrm{g}$ factor distribution.

Next, we compare the calculated results with the experimental ones. Figure 5(a) shows the calculated results about the components of $\langle\boldsymbol{S}\rangle$ and $\boldsymbol{B}_{\mathrm{n}}$ with the same condition in Fig. 3(a). In the figure, $\left\langle S_{y}\right\rangle$ and $B_{\mathrm{n}, y}$ are not shown for an easy view because of their small magnitudes within $\left|B_{x}\right|<\left|B_{x}^{\mathrm{c}}\right|$.

The calculations reproduce well the experimental results in Fig. 1 at the following characteristic points:

(1) a large value of $\left\langle S_{z}\right\rangle$ is preserved even under a large $\left|B_{x}\right|$ until the critical field $B_{x}^{\mathrm{c}}$,

(2) $\left\langle S_{z}\right\rangle$ changes abruptly at $B_{x}^{\mathrm{c}}$ and shows the hysteretic (thus, bistable) behavior,

(3) $\left\langle S_{z}\right\rangle$ curve is symmetric with respect to the sweep direction of $B_{x}$,

(4) $\left|B_{\mathrm{n}, z}\right|$ reduces gradually with increasing $\left|B_{x}\right|$.

The schematics of the electron spin polarization and the nuclear field are summarized in Fig. 5(b); the upper (lower) panel corresponds to the case $\left|B_{x}\right| \leqslant\left|B_{x}^{\mathrm{c}}\right|\left(\left|B_{x}\right|>\left|B_{x}^{\mathrm{c}}\right|\right)$. In the small- $B_{x}$ region, the in-plane component of $\boldsymbol{B}_{\mathrm{n}}$ compensates the applied field, and the effective field $\boldsymbol{B}_{\mathrm{T}}^{(\mathrm{e})}$ is almost parallel to the $z$ direction. Therefore, a large value of $\left\langle S_{z}\right\rangle$ is kept under the condition $\left|B_{x}\right| \leqslant\left|B_{x}^{\mathrm{c}}\right|$. In the large- $B_{x}$ region, on the other hand, since the induced $\boldsymbol{B}_{\mathrm{n}}$ is quite small, the vector $\langle\boldsymbol{S}\rangle$ feels a large transverse field and $\left\langle S_{z}\right\rangle$ relaxes quickly.

Note that the $B_{x}$ dependence of $r_{\mathrm{Q}}$ [as shown in Eq. (7)] is necessary to reproduce the gradual reduction in $B_{\mathrm{n}, z}$; if $r_{\mathrm{Q}}$ is independent of $B_{x}$ and has a constant value, the calculated $B_{\mathrm{n}, z}$ shows a similar shape with $\left\langle S_{z}\right\rangle$ and abrupt changes occur at $\left|B_{x}^{\mathrm{c}}\right|$. As shown in the lower panel of Fig. 5(a), small discontinuities at $\left|B_{x}^{\mathrm{c}}\right|$ arise in the model calculations, while the experimental results change continuously [Fig. 6(c)]. We guess tentatively that the step in Overhauser shift might be so small that we failed to detect it. Further, the tilting of $\boldsymbol{q}$ from the $z$ direction induces the horizontal shift of the Hanle curve. The outer $\left|B_{x}^{\mathrm{c}}\right|$ in Fig. 6(a) is slightly different depending on 


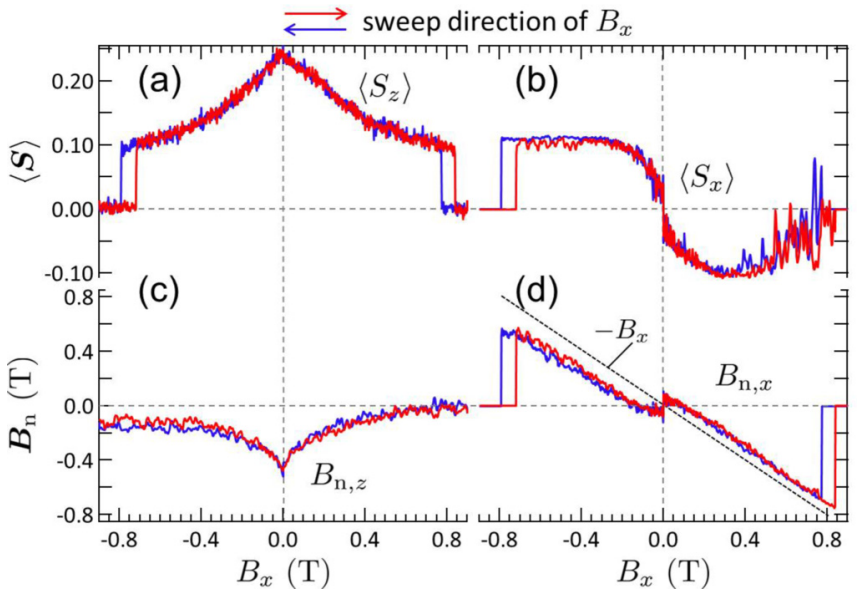

FIG. 6. (a) The observed $\left\langle S_{z}\right\rangle\left(=\rho_{\mathrm{c}} / 2\right)$, (b) the expected $\left\langle S_{x}\right\rangle$, (c) the observed $B_{\mathrm{n}, z}$, and (d) the expected $B_{\mathrm{n}, x}$. Red (blue) line represents the result with increasing (decreasing) $B_{x}$. (b) and (d) are obtained by substituting the observed $\left\langle S_{z}\right\rangle$ and $B_{\mathrm{n}, z}$ to Eqs. (B2) and (B3). The complete cancellation relation $B_{\mathrm{n}, x}=-B_{x}$ is indicated by a dotted line.

the sweep direction: $|-0.790| \mathrm{T}$ and $0.840 \mathrm{~T}$ in the blue and red curves, respectively. It may support the tilting of the $\boldsymbol{q}$ axis although further experimental investigations are required to deduce the tilting angle from the $z$ axis.

Finally, we provide the $B_{x}$ dependence of $\left\langle S_{x}\right\rangle$ and $B_{\mathrm{n}, x}$ expected from experimental data of $\left\langle S_{z}\right\rangle$ and $B_{\mathrm{n}, z}$ in Fig. 6. They are obtained from the steady state solutions of the Bloch equation (see Appendix B) by substituting the observed $\left\langle S_{z}\right\rangle=$ $\rho_{\mathrm{c}} / 2$ and $B_{\mathrm{n}, z}=\Delta E_{\mathrm{OHS}} /\left(g_{z}^{\mathrm{e}} \mu_{\mathrm{B}}\right)$ and solving for $\left\langle S_{x}\right\rangle$ and $B_{\mathrm{n}, x}$. Comparing the experimentally expected $\left|\left\langle S_{x}\right\rangle\right|$ [Fig. 6(b)] with the computed $\left|\left\langle S_{x}\right\rangle\right|$ [Fig. 5(a)], the expected one has the maximal value and shows saturation or decay while the computed $\left|\left\langle S_{x}\right\rangle\right|$ increases monotonically with increasing $\left|B_{x}\right|$. Asymmetry in the expected $\left|\left\langle S_{x}\right\rangle\right|$ with respect to $B_{x}$ is originated from the asymmetry of $B_{\mathrm{n}, z}$, which has small but finite value in a large negative $B_{x}$ region.

As a whole, the proposed model can reproduce the observed results qualitatively. However, the quantitative disagreement of the hysteresis width of $\left|B_{x}^{\mathrm{c}}\right|$ between the calculated and observed results could not be resolved at present in the range of the parameters we changed. Despite such a disagreement, $B_{\mathrm{n}, x}$ agrees well with the computed results. These suggest that there is a possibility that another mechanism for the in-plane nuclear field formation may work in a relatively large $\left|B_{x}\right|$ region. One plausible candidate is a noncollinear HFI process [29], which is not included in our proposed model. Although the main origin of the growth of $I_{x}$ is a flip-flop process between the finite $S_{x}$ and $I_{x}$, the cooperation of the noncollinear HFI (i.e., $\propto I_{x} S_{z}$ ) contributes to the formation of $I_{x}$ from $S_{z}$, and it may improve the qualitative agreement in the relatively large $B_{x}$ region. In addition, the introduction of the $B_{x}$-dependent $T_{\mathrm{ND}}$ may improve the quantitative features.

\section{CONCLUSIONS}

We investigated the in-plane nuclear field formation via Hanle effect measurements of $X^{+}$in single self-assembled
InAlAs quantum dots. The observed Hanle curves showed anomalously large full width and hysteretic behavior which cannot be explained by an existing model. To reproduce the measured anomalies, we proposed a phenomenological model including the nuclear quadrupolar effect and the sign inversion between the in-plane and out-of-plane nuclear $\mathrm{g}$ factors, which induced the compensation of $B_{x}$ by $B_{\mathrm{n}, x}$. The quadrupolar splitting allows a part of NSP to be preserved nearly along the $z$ axis, which induced the $\left\langle S_{x}\right\rangle$ that can change $I_{x}$ via collinear HFI. The proposed model reproduces well the characteristic points of the observed anomalous Hanle curve, and consequently it has the good qualitative agreement. Therefore, we conclude that the collinear HFI is a dominant mechanism for the in-plane nuclear field formation although the noncollinear HFI may contribute to the in-plane field formation in relatively large externally applied field.

\section{ACKNOWLEDGMENTS}

The authors would like to acknowledge H. Sasakura for sample growth and fruitful discussions. This work is supported by JSPS KAKENHI (Grants No. JP26800162 and No. JP17K19046) and the Asahi Glass Foundation (Japan).

\section{APPENDIX A: IN-PLANE ELECTRON G FACTORS}

Here, we evaluate the in-plane electron $\mathrm{g}$ factor, which is one of the key parameters to describe the Hanle curves and coupled electron-nuclear spin dynamics. Figure 7(a) shows the polarization-resolved PL spectra under nonpolarized excitation at $6 \mathrm{~K}$ and $0 \mathrm{~T}$ of a single InAlAs QD used in this study. The spectra indicate three emissions: the neutral biexciton $\left(X X^{0}\right)$, neutral exciton $\left(X^{0}\right)$, and positive trion $\left(X^{+}\right)$from the low energy side. Each charge state is assigned by considering the fine structure splitting (FSS) and the binding energy. The FSS of $\sim 73 \mu \mathrm{eV}$, the inverse pattern of FSS in the $X^{0}$ and $X X^{0}$ peaks, and no splitting in the $X^{+}$peak are observed clearly.

As shown in Fig. 7(b), a transverse magnetic field $B_{x}$ mixes the spin-up and spin-down states for the electron of $X^{+}$and hole, respectively, and the four transitions $\left(E_{1}, E_{2}, E_{3}, E_{4}\right)$ with linearly polarized emissions and absorptions become optically active. The Zeeman splittings of the outer peaks $\Delta E_{\text {out }}=\left|E_{1}-E_{4}\right|$ with $\pi^{x}$ polarization and of the inner peaks $\Delta E_{\text {in }}=\left|E_{2}-E_{3}\right|$ with $\pi^{y}$ polarization are given by $\Delta E_{\text {out }}=\left(\left|g_{x}^{\mathrm{e}}\right|+\left|g_{x}^{\mathrm{h}}\right|\right) \mu_{\mathrm{B}} B_{x}$ and $\Delta E_{\text {in }}=\left(\left|g_{x}^{\mathrm{e}}\right|-\left|g_{x}^{\mathrm{h}}\right|\right) \mu_{\mathrm{B}} B_{x}$, respectively. Here, $g_{x}^{\mathrm{h}}$ is the in-plane hole $\mathrm{g}$ factor. Figure $7(\mathrm{c})$ shows the polarization-resolved $X^{+}$PL spectra at $5 \mathrm{~T}$ under nonpolarized excitation. In this $\mathrm{QD}$, there is no splitting in $\pi^{y}$ PLs within our spectral resolution, and it indicates that the magnitude of $g_{x}^{\mathrm{e}}$ is very close to $g_{x}^{\mathrm{h}}$. Figure $7(\mathrm{~d})$ shows the observed energy splitting of $\pi^{x}$ PLs in a range of 2-5 T. The observed $\Delta E_{\text {out }}$ increases with $B_{x}$, and from the line fitting, the magnitudes of the electron and hole $\mathrm{g}$ factors are deduced to be $\left|g_{x}^{\mathrm{e}}\right| \simeq\left|g_{x}^{\mathrm{h}}\right|=0.35 \pm 0.01$. Moreover, the anisotropy of the in-plane electron $\mathrm{g}$ factor $\left|g_{\perp}^{\mathrm{e}}\right|$ was investigated by rotating the QD sample around the $z$ axis. Figure 7(e) is a polar plot of $\left|g_{\perp}^{\mathrm{e}}\right|$, and it indicates that the in-plane $\mathrm{g}$ factor anisotropy is negligible. In addition to the in-plane $\mathrm{g}$ factors, the electron and hole $\mathrm{g}$ factors in the $z$ direction of this QD were evaluated independently to be $g_{z}^{\mathrm{e}}=-0.34 \pm 0.02$ and $g_{z}^{\mathrm{h}}=2.57 \pm 0.01$ 
(a)

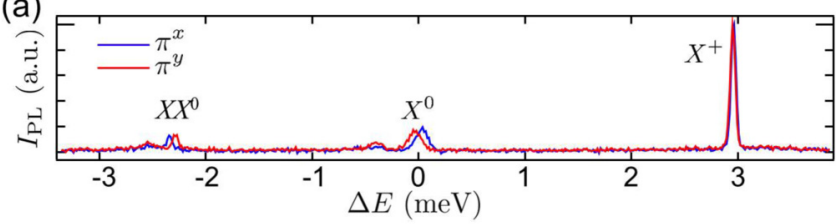

(b)

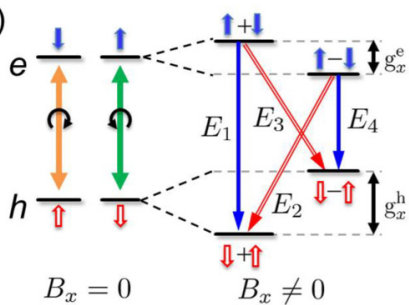

(c)

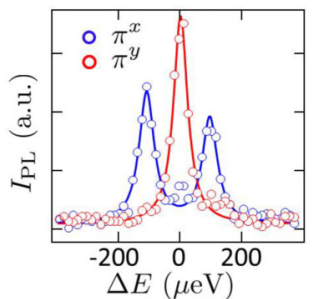

(d)

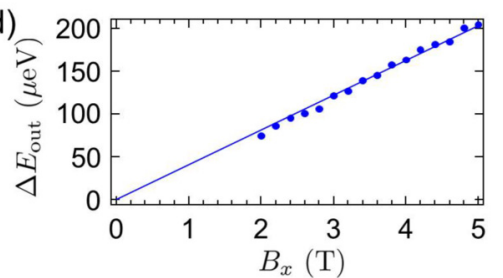

(e)

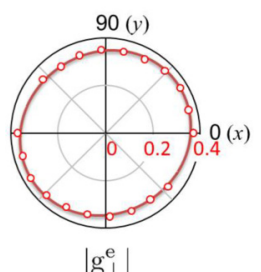

FIG. 7. (a) Polarization-resolved PL spectra of a typical SAInAlAs QD under nonpolarized excitation at $6 \mathrm{~K}$ and $0 \mathrm{~T}$. The horizontal axis is replotted from the midpoint of the $X^{0}$ doublet $(1.6419 \mathrm{eV})$. (b) A level diagram of the hole (open arrows) and electron (solid arrows) states under a transverse magnetic field $B_{x}$. (c) The $X^{+}$ PL spectra at $B_{x}=5 \mathrm{~T}$ detected in the $\left(\pi^{x}, \pi^{y}\right)$ basis. The origin of the horizontal axis is set to the energy of the inner PL peaks. (d) The observed Zeeman splitting of the outer peaks as a function of $B_{x}$. The solid line is a fitting curve. (e) Polar plot of the magnitude of in-plane electron $\mathrm{g}$ factor, $\left|g_{\perp}^{\mathrm{e}}\right|$.

by the method canceling an optically-induced nuclear magnetic field in the $z$ direction by a longitudinal field [22]. The obtained set of the electron $\mathrm{g}$ factor $\left(g_{x}^{\mathrm{e}}, g_{y}^{\mathrm{e}}, g_{z}^{\mathrm{e}}\right)$ is used in the section of model calculations.

\section{APPENDIX B: BLOCH EQUATION}

The steady state solutions of the Bloch equation [Eq. (1)] are written as

$$
\begin{aligned}
\langle\boldsymbol{S}\rangle= & \frac{S_{0}}{B_{1 / 2}^{2}+\left(B_{x}+B_{\mathrm{n}, x}\right)^{2}+B_{\mathrm{n}, y}^{2}+B_{\mathrm{n}, z}^{2}} \\
& \times\left[\begin{array}{c}
B_{1 / 2} B_{\mathrm{n}, y}+B_{\mathrm{n}, z}\left(B_{x}+B_{\mathrm{n}, x}\right) \\
B_{\mathrm{n}, y} B_{\mathrm{n}, z}-B_{1 / 2}\left(B_{x}+B_{\mathrm{n}, x}\right) \\
B_{1 / 2}^{2}+B_{\mathrm{n}, z}^{2}
\end{array}\right] .
\end{aligned}
$$

Omitting the component $B_{\mathrm{n}, y}$ which is very small in the simulated results, the $x$ and $z$ components of $\langle\boldsymbol{S}\rangle$ can be represented analytically as

$$
\begin{aligned}
& \left\langle S_{x}\right\rangle=S_{0} \frac{\left(B_{x}+B_{\mathrm{n}, x}\right) B_{\mathrm{n}, z}}{B_{1 / 2}^{2}+\left(B_{x}+B_{\mathrm{n}, x}\right)^{2}+B_{\mathrm{n}, z}^{2}}, \\
& \left\langle S_{z}\right\rangle=S_{0} \frac{B_{1 / 2}^{2}+B_{\mathrm{n}, z}^{2}}{B_{1 / 2}^{2}+\left(B_{x}+B_{\mathrm{n}, x}\right)^{2}+B_{\mathrm{n}, z}^{2}},
\end{aligned}
$$

where $B_{1 / 2}\left(=\hbar /\left(\left|g_{\perp}^{\mathrm{e}}\right| \mu_{\mathrm{B}} T_{\mathrm{S}}\right)\right)$ is a half width of the normal Lorentzian curve. Equations (B2) and (B3) were used to obtain the expected $\left\langle S_{x}\right\rangle$ and $B_{\mathrm{n}, x}$ from the observed $\left\langle S_{z}\right\rangle$ and $B_{\mathrm{n}, z}$ in Fig. 6.

\section{APPENDIX C: NUCLEAR QUADRUPOLE INTERACTION AND ZEEMAN INTERACTION FOR THE NUCLEI WITH $I=3 / 2$}

Assuming the axial symmetry, the nuclear quadrupole interaction Hamiltonian $\mathcal{H}_{\mathrm{Q}}=\hbar \omega_{\mathrm{Q}}\left[I_{q}^{2}-I(I+1) / 3\right] / 2[11]$ can be transformed to

$$
\begin{aligned}
\mathcal{H}_{\mathrm{Q}}= & \frac{\hbar \omega_{\mathrm{Q}}}{2}\left[I_{z}^{2} \cos ^{2} \theta_{\mathrm{Q}}-\frac{I(I+1)}{3}\right. \\
& \left.+\left(I_{z} I_{x}+I_{x} I_{z}\right) \sin \theta_{\mathrm{Q}} \cos \theta_{\mathrm{Q}}+I_{x}^{2} \sin ^{2} \theta_{\mathrm{Q}}\right]
\end{aligned}
$$

by using $I_{q}=I_{z} \cos \theta_{\mathrm{Q}}+I_{x} \sin \theta_{\mathrm{Q}}$, where $\theta_{\mathrm{Q}}$ is a tilting angle of the principal axis $\boldsymbol{q}$ from the growth axis $(z)$. Using a ladder operator $I_{x}=\left(I^{+}+I^{-}\right) / 2$, Eq. $(\mathrm{C} 1)$ can be written as

$$
\mathcal{H}_{\mathrm{Q}}=\frac{\hbar \omega_{\mathrm{Q}}}{2}(A+B+C),
$$

where

$$
\begin{aligned}
& A=I_{z}^{2} \cos ^{2} \theta_{\mathrm{Q}}-\frac{I(I+1)}{3}+\frac{1}{4}\left(I^{+} I^{-}+I^{-} I^{+}\right) \sin ^{2} \theta_{\mathrm{Q}} \\
& B=\frac{1}{2}\left(I_{z} I^{+}+I_{z} I^{-}+I^{+} I_{z}+I^{-} I_{z}\right) \sin \theta_{\mathrm{Q}} \cos \theta_{\mathrm{Q}} \\
& C=\frac{1}{4}\left[\left(I^{+}\right)^{2}+\left(I^{-}\right)^{2}\right] \sin ^{2} \theta_{\mathrm{Q}} .
\end{aligned}
$$

The $A, B$, and $C$ contribute to the diagonal term, transitions with $\Delta m_{z}= \pm 1$, and transitions with $\Delta m_{z}= \pm 2$, respectively.

In the case of the nuclei with $I=3 / 2$, the above Hamiltonian can be written as follows with the bases $\left|I, m_{z}\right\rangle=$ $\{|3 / 2,+3 / 2\rangle,|3 / 2,+1 / 2\rangle,|3 / 2,-1 / 2\rangle,|3 / 2,-3 / 2\rangle\}$,

$$
\mathcal{H}_{\mathrm{Q}}=\frac{\hbar \omega_{\mathrm{Q}}}{2}\left(\begin{array}{cccc}
A_{3 / 2} & B_{3 / 2} & C_{3 / 2} & 0 \\
B_{3 / 2} & D_{3 / 2} & 0 & C_{3 / 2} \\
C_{3 / 2} & 0 & D_{3 / 2} & -B_{3 / 2} \\
0 & C_{3 / 2} & -B_{3 / 2} & A_{3 / 2}
\end{array}\right) .
$$

Here

$$
\begin{aligned}
& A_{3 / 2}=\frac{9}{4} \cos ^{2} \theta_{\mathrm{Q}}-\frac{5}{4}+\frac{3}{4} \sin ^{2} \theta_{\mathrm{Q}} \\
& B_{3 / 2}=\sqrt{3} \sin \theta_{\mathrm{Q}} \cos \theta_{\mathrm{Q}}, \quad C_{3 / 2}=\frac{\sqrt{3}}{2} \sin ^{2} \theta_{\mathrm{Q}}, \\
& D_{3 / 2}=\frac{1}{4} \cos ^{2} \theta_{\mathrm{Q}}-\frac{5}{4}+\frac{7}{4} \sin ^{2} \theta_{\mathrm{Q}} .
\end{aligned}
$$

Similarly, assuming the axial symmetry, Zeeman interaction Hamiltonian can be given by

$$
\begin{aligned}
\mathcal{H}_{\mathrm{Z}} & =\frac{\hbar \omega_{\mathrm{Z}}}{2}\left(I_{z} \cos \theta_{\mathrm{B}}+I_{x} \sin \theta_{\mathrm{B}}\right) \\
& =\frac{\hbar \omega_{\mathrm{Z}}}{2}\left[I_{z} \cos \theta_{\mathrm{B}}+\left(I^{+}+I^{-}\right) \frac{\sin \theta_{\mathrm{B}}}{2}\right],
\end{aligned}
$$

where $\theta_{\mathrm{B}}$ is the angle of the applied field direction from the $z$ axis and $\hbar \omega_{\mathrm{Z}}$ is the Zeeman energy. The matrix representation 
is

$$
\mathcal{H}_{\mathrm{Z}}=\frac{\hbar \omega_{\mathrm{Z}}}{2}\left(\begin{array}{cccc}
a_{3 / 2} & b_{3 / 2} & 0 & 0 \\
b_{3 / 2} & c_{3 / 2} & d_{3 / 2} & 0 \\
0 & d_{3 / 2} & -c_{3 / 2} & b_{3 / 2} \\
0 & 0 & b_{3 / 2} & -a_{3 / 2}
\end{array}\right)
$$

where

$$
\begin{array}{ll}
a_{3 / 2} & =\frac{3}{2} \cos \theta_{\mathrm{B}}, \quad b_{3 / 2}=\frac{\sqrt{3}}{2} \sin \theta_{\mathrm{B}}, \\
c_{3 / 2} & =\frac{1}{2} \cos \theta_{\mathrm{B}}, \quad d_{3 / 2}=\sin \theta_{\mathrm{B}} .
\end{array}
$$

[1] Optical Orientation, Modern Problems in Condensed Matter Sciences Vol. 8, Chaps. 2 and 5, edited by F. Meier and B. Zakharchenya (North-Holland, New York, 1984).

[2] Spin Physics in Semiconductors, Springer Series in Solid-State Sciences Vol. 157, Chaps. 1 and 11, edited by M. I. Dyakonov (Springer, Berlin, 2008).

[3] Recent optical investigation of nuclear spin physics in QDs are reviewed comprehensively: B. Urbaszek, X. Marie, T. Amand, O. Krebs, P. Voisin, P. Maletinsky, A. Högele, and A. Imamoglu, Rev. Mod. Phys. 85, 79 (2013).

[4] D. Gammon, Al. L. Efros, T. A. Kennedy, M. Rosen, D. S. Katzer, D. Park, S. W. Brown, V. L. Korenev, and I. A. Merkulov, Phys. Rev. Lett. 86, 5176 (2001).

[5] T. Yokoi, S. Adachi, H. Sasakura, S. Muto, H. Z. Song, T. Usuki, and S. Hirose, Phys. Rev. B 71, 041307(R) (2005).

[6] B. Eble, O. Krebs, A. Lemaître, K. Kowalik, A. Kudelski, P. Voisin, B. Urbaszek, X. Marie, and T. Amand, Phys. Rev. B 74, 081306(R) (2006).

[7] P.-F. Braun, B. Urbaszek, T. Amand, X. Marie, O. Krebs, B. Eble, A. Lemaître, and P. Voisin, Phys. Rev. B 74, 245306 (2006).

[8] A. I. Tartakovskii, T. Wright, A. Russell, V. I. Fal'ko, A. B. Van'kov, J. Skiba-Szymanska, I. Drouzas, R. S. Kolodka, M. S. Skolnick, P. W. Fry, A. Tahraoui, H.-Y. Liu, and M. Hopkinson, Phys. Rev. Lett. 98, 026806 (2007).

[9] P. Maletinsky, C. W. Lai, A. Badolato, and A. Imamoglu, Phys. Rev. B 75, 035409 (2007).

[10] R. Kaji, S. Adachi, H. Sasakura, and S. Muto, Phys. Rev. B 77, 115345 (2008).

[11] C. P. Slichter, Principles of Magnetic Resonance, Chapter 10 (Springer, New York, 1996), 3rd ed.

[12] C. Latta, A. Högele, Y. Zhao, A. N. Vamivakas, P. Maletinsky, M. Kroner, J. Dreiser, I. Carusotto, A. Badolato, D. Schuh, W. Wegscheider, M. Atature, and A. Imamoglu, Nat. Phys. 5, 758 (2009).

[13] X. Xu, W. Yao, B. Sun, D. G. Steel, A. S. Bracker, D. Gammon, and L. J. Sham, Nature (London) 459, 1105 (2009).

[14] A. Högele, M. Kroner, C. Latta, M. Claassen, I. Carusotto, C. Bulutay, and A. Imamoglu, Phys. Rev. Lett. 108, 197403 (2012).

[15] O. Krebs, P. Maletinsky, T. Amand, B. Urbaszek, A. Lemaître, P. Voisin, X. Marie, and A. Imamoglu, Phys. Rev. Lett. 104, 056603 (2010).
[16] E. A. Chekhovich, K. V. Kavokin, J. Puebla, A. B. Krysa, M. Hopkinson, A. D. Andreev, A. M. Sanchez, R. Beanland, M. S. Skolnick, and A. I. Tartakovskii, Nat. Nanotech. 7, 646 (2012).

[17] E. A. Chekhovich, M. Hopkinson, M. S. Skolnick, and A. I. Tartakovskii, Nat. Commun. 6, 6348 (2015).

[18] R. I. Dzhioev and V. L. Korenev, Phys. Rev. Lett. 99, 037401 (2007).

[19] P. Maletinsky, doctoral thesis (Swiss Federal Institute of Technology, Zurich, 2008).

[20] J. Nilsson, L. Bouet, A. J. Bennett, T. Amand, R. M. Stevenson, I. Farrer, D. A. Ritchie, S. Kunz, X. Marie, A. J. Shields, and B. Urbaszek, Phys. Rev. B 88, 085306 (2013).

[21] G. Sallen, S. Kunz, T. Amand, L. Bouet, T. Kuroda, T. Mano, D. Paget, O. Krebs, X. Marie, K. Sakoda, and B. Urbaszek, Nat. Commun. 5, 3268 (2014).

[22] R. Matsusaki, R. Kaji, S. Yamamoto, H. Sasakura, and S. Adachi, arXiv:1703.06046.

[23] J. Debus, T. S. Shamirzaev, D. Dunker, V. F. Sapega, E. L. Ivchenko, D. R. Yakovlev, A. I. Toropov, and M. Bayer, Phys. Rev. B 90, 125431 (2014).

[24] H. Kumano, S. Kimura, M. Endo, H. Sasakura, S. Adachi, S. Muto, and I. Suemune, J. Nanoelectron. Optoelectron. 1, 39 (2006).

[25] R. Kaji, S. Adachi, H. Sasakura, and S. Muto, Phys. Rev. B 85, 155315 (2012).

[26] The following parameters are used in the calculations: $f_{\mathrm{e}}=$ $0.015, T_{\mathrm{ND}, k}=10 \mathrm{~ms}, B_{\mathrm{Q}}=280 \mathrm{mT}$, and isotropic $g_{k}^{\mathrm{e}}(k=$ $x, y, z)=-0.35$ are the measured values for this single $\mathrm{QD}$. Also, the following expected values are used: $\tau_{\mathrm{c}}=100 \mathrm{ps}$, $N=30,000, \tilde{A}_{x(y)}=-52.6 \mu \mathrm{eV}, \tilde{A}_{z}=+52.6 \mu \mathrm{eV}, g_{x(y)}^{\mathrm{n}}=$ -0.179 , and $g_{z}^{\mathrm{n}}=+0.179$. $r_{0}=1.0$, and $\boldsymbol{q}$ is assumed to be along the $z$ axis for simplicity.

[27] In the case of Faraday geometry, as the $B_{z}$ is increased, the state $|-3 / 2\rangle_{q}$ moves down in energy through a crossing with $|-1 / 2\rangle_{q}$ followed by a final anticrossing with $|+1 / 2\rangle_{q}$.

[28] M. S. Kuznetsova, R. V. Cherbunin, I. Ya. Gerlovin, I. V. Ignatiev, S. Yu. Verbin, D. R. Yakovlev, D. Reuter, A. D. Wieck, and M. Bayer, Phys. Rev. B 95, 155312 (2017).

[29] C.-W. Huang and X. Hu, Phys. Rev. B 81, 205304 (2010). 